MSC 49M05

\title{
A method for solving differential inclusions with fixed right end ${ }^{*}$
}

\section{A. V. Fominyh}

St. Petersburg State University, 7-9, Universitetskaya nab., St. Petersburg, 199034, Russian Federation

For citation: Fominyh A. V. A method for solving differential inclusions with fixed right end. Vestnik of Saint Petersburg University. Applied Mathematics. Computer Science. Control Processes, 2018, vol. 14, iss. 4, pp. 302-315. https://doi.org/10.21638/11702/spbu10.2018.403

In the paper, we study a differential inclusion with a given continuous convex multivalued mapping. For a given finite time interval, it is required to construct a solution of the differential inclusion, that satisfies the given initial condition or both the initial and final conditions. With the help of support functions, the original problem is reduced to the problem of global minimization of some functions in the space of piecewise continuous functions. In the case of continuous differentiability of the support function of a multivalued mapping with respect to the phase variables, this functional is Gateaux differentiable. In the paper, Gateaux gradient is found, necessary and (in some particular cases) sufficient conditions for the global minimum of the given functions are obtained. On the basis of these conditions, the method of steepest descent is applied to the original problem. Numerical examples illustrate the method realization.

Keywords: differential inclusions, support function, the steepest descent method.

1. Introduction. Differential inclusions $[1,2]$ are often necessary in mathematical modeling of systems with incomplete description $[3,4]$ and in analyzing the behavior of discontinuous systems [5]. It is also known, that many optimal control problems under natural assumptions may be reduced to differential inclusions [4]. Finding solutions for a differential inclusion is important for applications [6-8]. As a rule, it is possible to obtain an analytical solution of a differential inclusion only in special cases but in the other cases one has to use numerical methods for this purpose.

Well-known classical methods of ordinary differential equations have been applied to exploring Cauchy problem with differential inclusions (the forward Euler method [9], implicit Euler numerical scheme [10, 11], a modification of Euler scheme [12]). Some authors also applied Runge-Kutta schemes to discontinuous equations and differential inclusions (see, e. g., [13, 14]). Attainability sets of differential inclusions were also studied in some works (see, e. g., [15-18]). A survey of difference methods for differential inclusions can be found in [19]. A noticeably smaller number of papers is devoted to the problem of solving differential inclusions with fixed right end. For example, an algorithm to solve boundary value problems for differential inclusions was constructed in [20].

Although there exist many explorations of the problem considered in this article, papers with the constructive methods are just a quite small part of them. But even in these investigations, as it has been noted, the majority of the known numerical methods are applied to the Cauchy problem with differential inclusions. There are few works containing the case, when the right end is fixed. So this work is aimed to obtain concrete solutions (including problems with the fixed right). The general ideas of the method of the paper date back to the scientific school of V. F. Demyanov, which is distinguished by constructiveness

* The work is supported by Russian Found of Fundamental Research (project N 18-31-00014 mol-a).

(C) Санкт-Петербургский государственный университет, 2018 
of the developed methods. Another distinguishing feature of the methods of this scientific school (including the method of this article) is continuity in contrast to discrete methods in all the works (on numerical methods) listed above. Such an approach can sometimes be less effective in implementation and in computation (than its discrete analogs), but it is interesting theoretically (the method of this article is original in that it is not based on any discretization of the initial problem).

2. Statement of the problem. Consider the differential inclusion

$$
\dot{x} \in F(x, t)
$$

with the initial condition

$$
x(0)=x_{0} .
$$

In formula (1) $F(x, t)$ is the given continuous multivalued mapping, $t \in[0, T], x$ is a $n$-dimensional continuous vector-function of the phase coordinates with piecewise continuous (with the finite number of the points of discontinuity) and bounded derivative in the interval $[0, T], T>0$, is the given moment of time. In formula (2) $x_{0} \in R^{n}$ is the given vector. We assume, that the function $F(x, t)$ puts in correspondence a certain convex compact set from $R^{n}$ for every moment of time $t \in[0, T]$ and for every phase point $x \in R^{n}$. We also suppose, that the support function of the multivalued mapping $F(x, t)$ is differentiable in $x$ and that its derivative in $x$ is continuous. This assumption is satisfied, for example, in optimal control problems with controllable systems described by the ordinary differential equations system, where in the right-hand side there is a continuously differentiable function, and control belongs to some convex compact set for each $t \in[0, T]$.

It is required to find the vector-function $x^{*} \in C_{n}[0, T]$, which satisfies inclusion (1) at every point $t \in[0, T]$ and satisfies condition (2). (Actually, under the assumptions made, there exists even a continuously differentiable (classical) solution [4, p. 213, Corollary after Theorem 2].

Here $C_{n}[0, T]$ is the space of $n$-dimensional vector-functions, continuous in $[0, T]$, with derivative from the space $P_{n}[0, T], P_{n}[0, T]$ is the space of $n$-dimensional vectorfunctions, piecewise continuous and bounded in $[0, T]$, with the finite number of the points of discontinuity in the interval $[0, T]$. If $t_{0} \in[0, T)$ is the point of discontinuity of the vectorfunction $\dot{x}$, then we suppose, that $\dot{x}\left(t_{0}\right)$ is a right-handed derivative of the vector-function $\dot{x}$ at the point $t_{0}, \dot{x}(T)$ is a left-handed derivative of the vector-function $\dot{x}$ at the point $T$. For the arbitrary set $F \subset R^{n}$ define the support function (of the vector $\psi \in R^{n}$ ) as follows: $c(F, \psi)=\sup _{f \in F}\langle f, \psi\rangle$, where $\langle a, b\rangle$ is the inner product of the vectors $a, b \in R^{n}$.

Remark 1. Instead of trajectories from the space $C_{n}[0, T]$ with derivatives from the space $P_{n}[0, T]$ one can consider absolutely continuous in the interval $[0, T]$ trajectories with measurable and almost everywhere bounded in $[0, T]$ derivatives (which must satisfy the differential inclusion almost everywhere on $[0, T]$ ) respectively. The choice of the space of solutions in the paper is explained by the possibility of their practical construction. Note, that in the Cauchy problem there usually exists infinite number of solutions. As it has been noted, this set of solutions contains a continuously differentiable solution. But as we will see below, there is no guarantee, that we will obtain such a solution, applying the method of the paper (we will possibly obtain a solution, which is not continuously differentiable (see examples below, point 7)).

3. Equivalent statement of the problem. Further, for brevity, we sometimes write $F$ instead of $F(x, t)$. Since for all $t \in[0, T]$ and for all $x \in R^{n}$ the multivalued mapping $F(x, t)$ is a convex, closed and bounded set in $R^{n}$, inclusion (1) may be rewritten as follows [3]: 


$$
\langle\dot{x}(t), \psi\rangle \leqslant c(F(x(t), t), \psi) \quad \forall \psi \in S, \quad \forall t \in[0, T],
$$

where $S$ is the unit sphere in $R^{n}$ with the center in the origin.

Denote $z(t)=\dot{x}(t), z \in P_{n}[0, T]$, then from (2), we get

$$
x(t)=x_{0}+\int_{0}^{t} z(\tau) d \tau .
$$

Put

$$
\begin{gathered}
\ell(\psi, z, t)=\langle z(t), \psi\rangle-c(F(x(t), t), \psi), \\
h(z, t)=\max _{\psi \in S} \max \{0, \ell(\psi, z, t)\}
\end{gathered}
$$

and construct the functional

$$
\varphi(z)=\frac{1}{2} \int_{0}^{T} h^{2}(z(t), t) d t .
$$

Consider the set

$$
\Omega=\left\{z \in P_{n}[0, T] \mid \varphi(z)=0\right\} .
$$

It is not difficult to see, that for functional (5) the relations are valid

$$
\begin{gathered}
\varphi(z)=0(z \in \Omega) \text {, if }\langle\dot{x}(t), \psi\rangle \leqslant c(F(x(t), t), \psi) \quad \forall \psi \in S, \quad \forall t \in[0, T], \\
\varphi(z)>0(z \notin \Omega) \text {, otherwise. }
\end{gathered}
$$

So, the problem of finding a solution of differential inclusion (1) with initial condition (2) is reduced to the minimization of functional (5) in the space $P_{n}[0, T]$. If $z^{*} \in P_{n}[0, T]$ is the solution of this problem, then

$$
x^{*}(t)=x_{0}+\int_{0}^{t} z^{*}(\tau) d \tau
$$

is the solution of problem (1), (2).

4. Differential properties of functional $\varphi$. As it has been noted, we suppose, that the support function $c(F(x, t), \psi)$ of the multivalued mapping $F(x, t)$ is differentiable in the phase variable $x$ and that the vector-function $\frac{\partial c(F(x, t), \psi)}{\partial x}$ is continuous in $(x, \psi, t)$. Then for all $x, y \in C_{n}[0, T]$, and for all $\psi \in S, t \in[0, T]$, the following relation holds:

$$
\begin{gathered}
c(F(x(t)+\alpha y(t), t), \psi)-c(F(x(t), t), \psi)= \\
=\alpha\left\langle\frac{\partial c(F(x(t), t), \psi)}{\partial x}, y(t)\right\rangle+o(\alpha, x(t), y(t), \psi, t), \\
\frac{o(\alpha, x(t), y(t), \psi, t)}{\alpha} \rightarrow 0, \text { when } \alpha \downarrow 0 .
\end{gathered}
$$

Let $v \in P_{n}[0, T]$. Fix the point $z \in P_{n}[0, T]$. Put

$$
z_{\alpha}(t)=z(t)+\alpha v(t)
$$




$$
y(t)=\int_{0}^{t} v(\tau) d \tau
$$

Using the property of the additivity of a support function in the first argument [4, p. 198, Property 3] and equations (6), (7), let us calculate

$$
\begin{gathered}
\ell\left(\psi, z_{\alpha}, t\right)=\ell(\psi, z, t)+\alpha H_{1}(\psi, z, v, t)+o(\alpha, \psi, t), \\
\frac{o(\alpha, \psi, t)}{\alpha} \rightarrow 0, \text { when } \alpha \downarrow 0,
\end{gathered}
$$

where

$$
H_{1}(\psi, z, v, t)=\langle\psi, v(t)\rangle-\left\langle\int_{0}^{t} v(\tau) d \tau, \frac{\partial c(F(x(t), t), \psi)}{\partial x}\right\rangle .
$$

Using relations (3), (4), find

$$
\begin{gathered}
h\left(z_{\alpha}, t\right)=h(z, t)+\alpha H(z, v, t)+o(\alpha, t), \\
\frac{o(\alpha, t)}{\alpha} \rightarrow 0, \text { when } \alpha \downarrow 0,
\end{gathered}
$$

here

$$
\begin{gathered}
H(z, v, t)=\max _{\psi \in \bar{R}(z, t)} H_{1}(\psi, z, v, t), \text { if } \max _{\psi \in S} \ell(\psi, z, t)>0, \\
H(z, v, t)=0, \text { if } \max _{\psi \in S} \ell(\psi, z, t)<0, \\
H(z, v, t)=\max _{\psi \in \bar{R}(z, t)} \max \left\{0, H_{1}(\psi, z, v, t)\right\}, \text { if } \max _{\psi \in S} \ell(\psi, z, t)=0,
\end{gathered}
$$

with

$$
\bar{R}(z, t)=\left\{\bar{\psi}(z, t) \in S \mid \max \{0, \ell(\bar{\psi}, z, t)\}=\max _{\psi(t) \in S} \max \{0, \ell(\psi, z, t)\}\right\} .
$$

Due to the structure of functional (3) it is easy to see, that in the case $\ell(\psi, z, t)>0$ the maximum of the function

$$
\max \{0, \ell(\psi, z, t)\}=\ell(\psi, z, t)
$$

is reached at the only element $\psi^{*}(z, t) \in S$. Actually, in this case the point $z$ does not belong to the set $F$. From the properties of the support function, it is known [3], that at every fixed $t \in[0, T]$ the value $h(z(t), t)$ is the Euclidean distance from the point $z(t)$ to the set $F(x(t), t)$, that is $h(z(t), t)=\|z(t)-f(x(t), t)\|$ (the Euclidean norm), where $f(x(t), t)$ is the projection of the point $z(t)$ on the set $F(x(t), t)$, which is unique as $F(x(t), t)$ is the convex compact. Hence it is clear, that the maximum of the expression $h(z(t), t)=\ell(\psi, z(t), t)=\langle z(t), \psi\rangle-c(F(x(t), t), \psi)=\|z(t)-f(x(t), t)\|$ is reached at the vector $\psi^{*}(z(t), t)=(z(t)-f(x(t), t)) /\|z(t)-f(x(t), t)\|$, which is unique because of the uniqueness of the vector $f(x(t), t)$. So in this case the set $\bar{R}(z, t)$ consists of the only element $\psi^{*}(z, t)$.

Now, it is not difficult to obtain the expansion

$$
\varphi\left(z_{\alpha}\right)=\varphi(z)+\alpha \int_{0}^{T} h(z(t), t) H(z(t), v(t), t)+o(\alpha, t) d t .
$$


It is obvious, that if $z \in \Omega$, then the functional $\varphi$ is Gateaux differentiable, and its Gateaux gradient vanishes.

Consider the case $z \notin \Omega$. Denote

$$
w(z, t)=\max _{\psi \in S} \ell(\psi, z, t)^{2}>0
$$

As it has been already noted, in this case this maximum is reached at the only element $\psi^{*}(z, t)$. Then the function $\psi^{*}(z, t)$ is continuous in $z$ at the fixed $t$ [21], therefore, in view of the continuity of the function $\frac{\partial c(F(x, t), \psi)}{\partial x}$ we conclude, that the function $\frac{\partial w(z, t)}{\partial z}$ is continuous in $z$ at the fixed $t$. Further, by Lagrange's mean value theorem there exists such a number $\theta=\theta(t) \in[0,1]$, that

$$
\begin{gathered}
w(z+\alpha v, t)-w(z, t)=\alpha\left\langle\frac{\partial w(z+\theta \alpha v, t)}{\partial z}, v\right\rangle= \\
=\alpha\left\langle\frac{\partial w(z, t)}{\partial z}, v\right\rangle+\alpha\left\langle\frac{\partial w(z+\theta \alpha v, t)}{\partial z}-\frac{\partial w(z, t)}{\partial z}, v\right\rangle \quad \forall t \in[0, T] .
\end{gathered}
$$

Then we have

$$
\begin{aligned}
& \left|\frac{\varphi(z+\alpha v)-\varphi(z)}{\alpha}-\int_{0}^{T}\left\langle v(t), \frac{\partial w(z(t), t)}{\partial z}\right\rangle d t\right|= \\
= & \left|\int_{0}^{T}\left\langle v(t), \frac{\partial w(z(t)+\theta \alpha v(t), t)}{\partial z}-\frac{\partial w(z(t), t)}{\partial z}\right\rangle d t\right| \leqslant \\
\leqslant & \max _{t \in[0, T]}\|v(t)\| \int_{0}^{T}\left\|\frac{\partial w(z(t)+\theta \alpha v(t), t)}{\partial z}-\frac{\partial w(z(t), t)}{\partial z}\right\| d t .
\end{aligned}
$$

The multiplier $\max _{t \in[0, T]}\|v(t)\|$ is bounded, as $v \in P_{n}[0, T]$. The norm under the integral tends to zero, when $\alpha \downarrow 0$ at the every fixed $t \in[0, T]$ due to the continuity of the function $\frac{\partial w(z, t)}{\partial z}$ in $z$ at the fixed $t$. Besides, this norm is bounded due to the continuity of the function $\frac{\partial c(F(x, t), \psi)}{\partial x}$. Then by Lebesgue's dominated convergence theorem expression (9) tends to zero, when $\alpha \downarrow 0$, what proves Gateaux differentiability of the functional $\varphi$ in the case $z \notin \Omega$.

Theorem 1. If the support function $c(F, \psi)$ of the multivalued mapping $F(x, t)$ is differentiable in the phase variable $x$ and its derivative in $x$ is continuous, then the functional $\varphi$ is Gateaux differentiable and its gradient at the point $z$ is expressed by the formula

$$
\nabla \varphi(z)=h(z, t) \psi^{*}(z, t)-\int_{t}^{T} h(z(\tau), \tau) \frac{\partial c\left(F(x(\tau), \tau), \psi^{*}(z(\tau), \tau)\right)}{\partial x} d \tau
$$

where $\psi^{*}(z, t) \in \bar{R}(z, t)$.

5. Necessary minimum conditions. From the known minimum condition $[22$, p. 85, Theorem 2.5.1] of Gateaux differentiable functional, expression (10) and the uniqueness of the zero solution of the homogeneous Volterra integral equation of the second kind we conclude, that the following theorem holds. 
Theorem 2. Let the conditions of Theorem 1 hold. For the point $x^{*}$ to satisfy inclusion (1) and condition (2), it is necessary and sufficient to have

$$
h\left(z^{*}, t\right) \psi^{*}\left(z^{*}, t\right)-\int_{t}^{T} h\left(z^{*}(\tau), \tau\right) \frac{\partial c\left(F\left(x^{*}(\tau), \tau\right), \psi^{*}\left(z^{*}(\tau), \tau\right)\right)}{\partial x} d \tau=0_{n},
$$

where $\psi^{*}\left(z^{*}, t\right) \in \bar{R}\left(z^{*}, t\right)$, and $0_{n}$ is the zero element of the space $P_{n}[0, T]$.

Let us return to the original problem. Suppose, in addition to initial condition (2), there is a finite restriction on the trajectory

$$
x(T)=x_{T},
$$

here $x_{T} \in R^{n}$ is the given vector.

It is required to find the vector-function $x^{*} \in C_{n}[0, T]$, which is a solution of differential inclusion (1) and satisfies boundary conditions (2), (12). Suppose, that there exists such a solution.

Introduce the functional

$$
\begin{gathered}
I(z)=\frac{1}{2}\left[\int_{0}^{T} h^{2}(z(t), t) d t+\left(x_{0}+\int_{0}^{T} z(t) d t-x_{T}\right)^{2}\right]= \\
=\varphi(z)+\chi(z) .
\end{gathered}
$$

It is obvious, that the problem is reduced to the minimization of functional (13) in the space $P_{n}[0, T]$. If $z^{*} \in P_{n}[0, T]$ is the solution of this problem, then

$$
x^{*}(t)=x_{0}+\int_{0}^{t} z^{*}(\tau) d \tau
$$

is the solution of problem (1), (2), (12).

Using again expansion (8), and also calculating Gateaux gradient for the functional $\chi$ and using the known minimum condition [22, p. 85, Theorem 2.5.1], we verify the following theorem.

Theorem 3. Let the conditions of Theorem 1 hold. For the point $x^{*}$ to satisfy inclusion (1) and conditions (2), (12), it is necessary to have

$$
\begin{gathered}
\nabla \varphi\left(z^{*}\right)+\nabla \chi\left(z^{*}\right)= \\
=h\left(z^{*}, t\right) \psi^{*}\left(z^{*}, t\right)-\int_{t}^{T} h\left(z^{*}(\tau), \tau\right) \frac{\partial c\left(F\left(x^{*}(\tau), \tau\right), \psi^{*}\left(z^{*}(\tau), \tau\right)\right)}{\partial x} d \tau+ \\
+x_{0}+\int_{0}^{T} z^{*}(t) d t-x_{T}=0_{n},
\end{gathered}
$$

where $\psi^{*}\left(z^{*}, t\right) \in \bar{R}\left(z^{*}, t\right)$.

If we have $I\left(z^{*}\right)=0$, then condition (14) is also sufficient.

6. The steepest descent method. Describe the steepest descent method [23] for finding stationary points of the functional $\varphi$ (the functional $I$ ). Fix an arbitrary point 
$z_{1} \in P_{n}[0, T]$. Let the point $z_{k} \in P_{n}[0, T]$ be already constructed. If minimum condition (11) or (14) holds, then the point $z_{k}$ is the stationary point of the functional $\varphi$ (the functional $I$ ), and the process terminates. Otherwise put

$$
\begin{gathered}
z_{k+1}=z_{k}-\gamma_{k} \nabla \varphi\left(z_{k}\right) \\
\left(z_{k+1}=z_{k}-\gamma_{k} \nabla I\left(z_{k}\right)\right),
\end{gathered}
$$

here the vector-function

$$
x_{k}(t)=x_{0}+\int_{0}^{t} z_{k}(\tau) d \tau,
$$

and the magnitude $\gamma_{k}$ is the solution of the following one-dimensional minimization problem:

$$
\begin{aligned}
\min _{\gamma>0} \varphi\left(z_{k}-\gamma \nabla \varphi\left(z_{k}\right)\right) & =\varphi\left(z_{k}-\gamma_{k} \nabla \varphi\left(z_{k}\right)\right) \\
\left(\min _{\gamma>0} I\left(z_{k}-\gamma \nabla I\left(z_{k}\right)\right)\right. & \left.=I\left(z_{k}-\gamma_{k} \nabla I\left(z_{k}\right)\right)\right) .
\end{aligned}
$$

Due to $(15) \varphi\left(z_{k+1}\right) \leqslant \varphi\left(z_{k}\right)\left(I\left(z_{k+1}\right) \leqslant I\left(z_{k}\right)\right)$. If the sequence $\left\{z_{k}\right\}$ is finite, then its last point is the stationary point of the functional $\varphi$ (the functional $I$ ) by construction.

Let the functional $\nabla \varphi$ (the functional $\nabla I$ ) be Lipschitz continuous on the ball in $L_{n}^{2}[0, T]$ with the center in the origin and the radius $\bar{r}>\sup _{z \in Z_{1}}\|z\|_{L_{n}^{2}[0, T]}$. (Lebesgue set $Z_{1}=\left\{z \in P_{n}[0, T] \mid \varphi(z) \leqslant \varphi\left(z_{1}\right)\left(I(z) \leqslant I\left(z_{1}\right)\right)\right\}$ is supposed to be bounded in the $L_{n}^{2}[0, T]$ norm.) If the sequence $\left\{z_{k}\right\}$ is infinite, then it can be shown, that the method converges [23] in the sense

$$
\begin{gathered}
\left\|\nabla \varphi\left(z_{k}\right)\right\|_{L_{n}^{2}[0, T]} \rightarrow 0, \text { when } k \rightarrow \infty \\
\left(\left\|\nabla I\left(z_{k}\right)\right\|_{L_{n}^{2}[0, T]} \rightarrow 0, \text { when } k \rightarrow \infty\right),
\end{gathered}
$$

where $L_{n}^{2}[0, T]$ is the space of square summable $n$-dimensional vector-functions in the interval $[0, T]$.

7. Numerical examples. Let us consider some examples of the implementation of the proposed algorithm. In the first three examples, differential inclusions with a free right end are investigated, then in the following two examples the right end is fixed. The symbol' means transposition. The calculations were performed symbolically in the Maple 12.0 package.

Example 1. Consider the differential inclusion

$$
\dot{x} \in F(x), \quad F(x)=\left([-1,1], x_{1}\right)^{\prime}, \quad t \in[0,1],
$$

and the initial condition

$$
x(0)=(0,0)^{\prime} .
$$

Here one of the solutions is evident. For example, one can take $z_{1}=\beta \in[-1,1]$, then $x_{1}=\beta t, z_{2}=x_{1}=\beta t$ and $x_{2}=0.5 \beta t^{2}$. However, let us demonstrate in detail the steps of the algorithm in this simple example. We choose a starting point, which is not a solution, and make one iteration according to the described algorithm. 
In this example $c(F, \psi)=\left|\psi_{1}\right|+x_{1} \psi_{2}, \quad \frac{\partial c}{\partial x}=\left(\psi_{2}, 0\right)^{\prime}$. Put $z_{1}=\left(2, x_{1}{ }_{-1}\right)^{\prime}$, where $x_{1}{ }_{-}$ is $x_{1}$ on the first iteration.

By formula (3) we have

$$
\begin{gathered}
\ell\left(\psi, z_{1}, t\right)=2 \psi_{1}-\left|\psi_{1}\right|=\psi_{1}, \quad \psi_{1} \geqslant 0, \\
\ell\left(\psi, z_{1}, t\right)=3 \psi_{1}, \quad \psi_{1}<0,
\end{gathered}
$$

and

$$
\max _{\psi \in S}\left(2 \psi_{1}-\left|\psi_{1}\right|\right)=1, \text { where } \psi^{*}=(1,0)^{\prime},
$$

and from (4) we get

$$
h\left(z_{1}, t\right)=1 .
$$

The gradient of the functional $\varphi$ at the point $z_{1}$ is of the form (see (10))

$$
\nabla \varphi\left(z_{1}\right)=(1,0)^{\prime}-\int_{t}^{1}(0,0)^{\prime} d \tau=(1,0)^{\prime} .
$$

Construct the next point $z_{2}=\left(2-\gamma, x_{2}{ }_{1}\right)^{\prime}$, where $x_{2_{-} 1}$ is $x_{1}$ on the second iteration.

Calculate

$$
\begin{gathered}
\ell\left(\psi, z_{2}, t\right)=(2-\gamma) \psi_{1}-\left|\psi_{1}\right|=(1-\gamma) \psi_{1}, \quad \psi_{1} \geqslant 0, \\
\ell\left(\psi, z_{2}, t\right)=(3-\gamma) \psi_{1}, \quad \psi_{1}<0 .
\end{gathered}
$$

For the point $z_{2}$ to satisfy the considered differential inclusion, it is necessary to have $\ell\left(\psi, z_{2}, t\right) \leqslant 0$ for all $\psi \in S$ and for all $t \in[0, T]$, which is true, when $\gamma_{1} \in[1,3]$.

We get $z_{2}=\left(2-\gamma_{1}, x_{2 \_}\right)=\left(\beta, x_{2}{ }_{-}\right), \beta \in[-1,1]$, so the point on the second iteration

$$
x^{*}(t):=x_{2}(t)=\int_{0}^{t} z_{2}(\tau) d \tau
$$

is the solution of this example.

Example 2. Consider the differential inclusion

$$
\dot{x} \in F(x, t), \quad F(x, t)=[0.5 x t, 1.5 x t], \quad t \in[0,1],
$$

and the initial condition

$$
x(0)=1 .
$$

In this example $c(F, \psi)=x t(\psi+0.5|\psi|), \frac{\partial c}{\partial x}=t(\psi+0.5|\psi|)$, if $x>0$. We see, that here appears an additional phase restriction, so formally the considered method can't be applied to this problem (as in the statement of the problem considered in this paper, we don't have the phase constraints). However, the method was successfully applied to this problem, as with the chosen point $z_{1}$ the points $x_{i}$ satisfy the constraint $x_{i}>0$ at all iterations.

Put $z_{1}=1$, then $x_{1}=t+1$. By formula (3) we have

$$
\begin{aligned}
& \ell\left(\psi, z_{1}, t\right)=\psi-t(t+1)(\psi+0.5|\psi|)=\psi-1.5 t(t+1) \psi, \quad \psi \geqslant 0, \\
& \ell\left(\psi, z_{1}, t\right)=\psi-t(t+1)(\psi+0.5|\psi|)=\psi-0.5 t(t+1) \psi, \quad \psi<0,
\end{aligned}
$$


and

$$
\max _{\psi \in S}(\psi-t(t+1)(\psi+0.5|\psi|))=1-1.5 t(t+1), \quad t \in[0,1], \quad \psi^{*}=1 .
$$

Let $t^{*} \in[0,1]$ be the root of the equation $1-1.5 t(t+1)=0$, and from (4) we get

$$
\begin{gathered}
h\left(z_{1}, t\right)=1-1.5 t(t+1), \quad t \in\left[0, t^{*}\right], \\
h\left(z_{1}, t\right)=0, \quad t \in\left[t^{*}, 1\right] .
\end{gathered}
$$

The gradient of the functional $\varphi$ at the point $z_{1}$ is of the form (see (3))

$$
\begin{gathered}
\nabla \varphi\left(z_{1}\right)=1-1.5 t(t+1)-\int_{t}^{t^{*}}(1-1.5 \tau(\tau+1)) 1.5 \tau d \tau, \quad t \in\left[0, t^{*}\right], \\
\nabla \varphi\left(z_{1}\right)=0_{n}, \quad t \in\left[t^{*}, 1\right] .
\end{gathered}
$$

Applying the algorithm, one can see, that the point on the 3rd iteration (with precision up to the 4th sign)

$$
x^{*}(t):=x_{3}=\left\{\begin{array}{l}
0.5837 t^{5}-0.5086 t^{4}+0.3914 t^{3}+ \\
+0.6187 t^{2}+0.0107 t+1, \quad t \in\left[0, t^{*}\right], \\
1.1612+0.5 t^{2}, \quad t \in\left[t^{*}, 1\right]
\end{array}\right.
$$

is the solution of this example. Figure 1 shows the functions $0.5 x^{*}(t) t, 1.5 x^{*}(t) t$ and $z^{*}(t)$ (on the 3-rd iteration). As the function $z^{*}(t)$ lies "between" the functions $0.5 x^{*}(t) t$ and $1.5 x^{*}(t) t$ at every $t \in[0,1]$, we see, that inclusion (16) is satisfied. (The condition (17) is automatically satisfied at every iteration.)

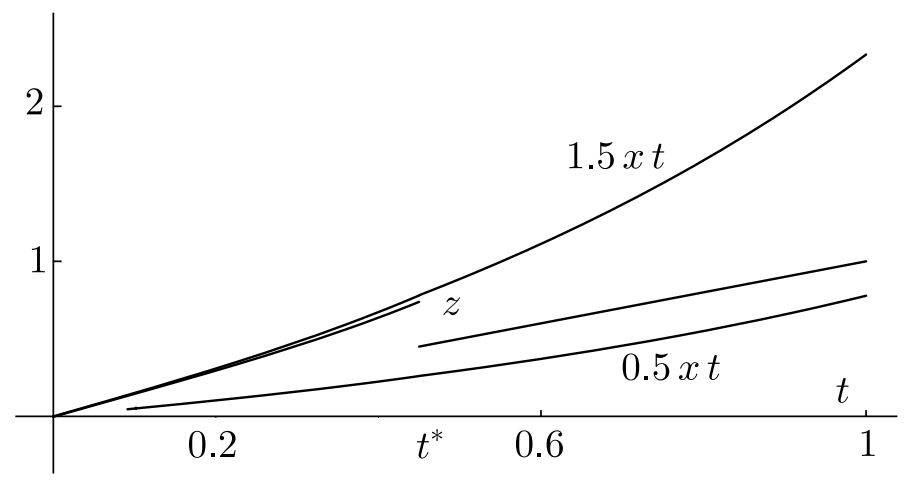

Figure 1. Solution of Example 2

Example 3. Consider the differential inclusion

$$
\dot{x} \in F(x), \quad F(x)=\left(x_{2}, x_{1}\right)^{\prime}+B, \quad t \in[0,1],
$$

where $B$ is a unit ball in $R^{n}$ with the center in the origin $\left(\left\{B=\left\{x \in R^{2} \mid \sum_{i=1}^{2} x_{i}^{2} \leqslant 1\right\}\right)\right.$, and the boundary conditions

$$
x(0)=(0.5,0.25)^{\prime},
$$




$$
x(1)=(1.75,2)^{\prime} .
$$

In this example $c(F, \psi)=x_{2} \psi_{1}+x_{1} \psi_{2}+\sqrt{\psi_{1}^{2}+\psi_{2}^{2}}, \frac{\partial c}{\partial x}=\left(\psi_{2}, \psi_{1}\right)^{\prime}$. Put $z_{1}=(2,1)^{\prime}$, then $x_{1}=(0.5+2 t, 0.25+t)^{\prime}$.

By formula (3) we have

$$
\begin{gathered}
\ell\left(\psi, z_{1}, t\right)=2 \psi_{1}+\psi_{2}-(0.25+t) \psi_{1}-(0.5+2 t) \psi_{2}-\sqrt{\psi_{1}^{2}+\psi_{2}^{2}}= \\
=(1.75-t) \psi_{1}+(0.5-2 t) \psi_{2}-\sqrt{\psi_{1}^{2}+\psi_{2}^{2}}, \\
\max _{\psi \in S}\left((1.75-t) \psi_{1}+(0.5-2 t) \psi_{2}-\sqrt{\psi_{1}^{2}+\psi_{2}^{2}}\right)= \\
=\sqrt{(1.75-t)^{2}+(0.5-2 t)^{2}}-1
\end{gathered}
$$

where

$$
\psi^{*}=\left(\frac{1.75-t}{\sqrt{(1.75-t)^{2}+(0.5-2 t)^{2}}}, \frac{0.5-2 t}{\sqrt{(1.75-t)^{2}+(0.5-2 t)^{2}}}\right)^{\prime},
$$

and from (4) we get

$$
h\left(z_{1}, t\right)=\sqrt{(1.75-t)^{2}+(0.5-2 t)^{2}}-1 .
$$

The gradient of the functional $\varphi$ at the point $z_{1}$ is of the form (see (10))

$$
\begin{aligned}
& \nabla \varphi\left(z_{1}\right)=\frac{h\left(z_{1}, t\right)}{\sqrt{(1.75-t)^{2}+(0.5-2 t)^{2}}}(1.75-t, 0.5-2 t)^{\prime}- \\
& -\int_{t}^{1} \frac{h\left(z_{1}, \tau\right)}{\sqrt{(1.75-\tau)^{2}+(0.5-2 \tau)^{2}}}(0.5-2 \tau, 1.75-\tau)^{\prime} d \tau .
\end{aligned}
$$

Applying the algorithm, one can see, that the point on the 4th iteration (with precision up to the 4th sign)

$$
\begin{aligned}
& x_{1}^{*}(t):=x_{4_{-} 1}=\left\{\begin{array}{l}
0.5-0.0525 t^{3}+0.4248 t^{2}+ \\
+0.7192 t+0.0078 t^{4}-0.001 t^{5}, \quad t \in[0,0.1021], \\
0.5008-0.1707 t^{3}+0.7395 t^{2}+0.6804 t, \quad t \in[0.1021,1],
\end{array}\right. \\
& x_{2}^{*}(t):=x_{4}{ }_{-2}=\left\{\begin{array}{l}
0.25+0.1424 t^{3}-0.0808 t^{2}+ \\
+1.3539 t-0.0022 t^{4}+0.0012 t^{5}, \quad t \in[0,0.1021], \\
0.2630-0.1027 t^{3}+ \\
+0.6897 t^{2}+1.1499 t, \quad t \in[0.1021,1],
\end{array}\right.
\end{aligned}
$$

is the solution of this example. (The first index denotes the iteration number, and the second index denotes the coordinate number.)

Rewrite inclusion (18) in the following way:

$$
\begin{aligned}
& \dot{x}_{1}(t)=x_{2}(t)+u_{1}(t), \\
& \dot{x}_{2}(t)=x_{1}(t)+u_{2}(t),
\end{aligned}
$$




$$
\sqrt{u_{1}^{2}(t)+u_{2}^{2}(t)} \leqslant 1, \quad t \in[0,1] .
$$

Figure 2 shows the functions $x_{1}^{*}(t)$ and $x_{2}^{*}(t)$ (on the 4 -th iteration). As we can see, condition $(20)$ is satisfied. Figure 2 also shows the function $\sqrt{u_{1}^{* 2}(t)+u_{2}^{* 2}(t)}$, where $u_{1}^{*}(t)=$ $z_{1}^{*}(t)-x_{2}^{*}(t), u_{2}^{*}(t)=z_{2}^{*}(t)-x_{1}^{*}(t)$ (on the 4-th iteration). As $\sqrt{u_{1}^{* 2}(t)+u_{2}^{* 2}(t)}$ $\leqslant 1$ at every $t \in[0,1]$, we see, that inclusion (18) is satisfied. (Condition (19) is automatically satisfied at every iteration.)
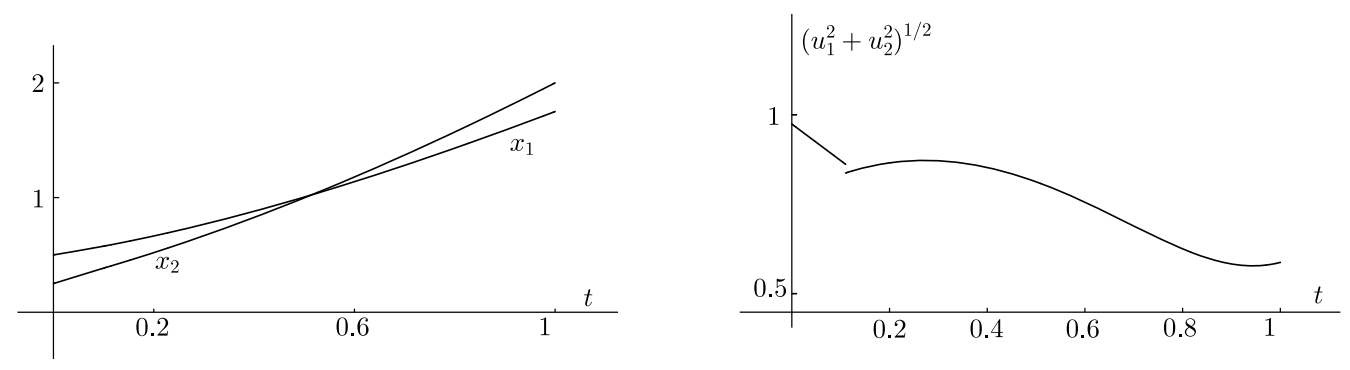

Figure 2. Solution of Example 3

Example 4. Consider the differential inclusion

$$
\dot{x} \in F(x), \quad F(x)=\left(x_{2}^{2}, 2 x_{1}\right)^{\prime}+D, \quad t \in[0,1],
$$

where $D$ is a unit rhombus $R^{n}$ with the center in the origin $\left(D=\left\{x \in R^{2}\left|\sum_{i=1}^{2}\right| x_{i} \mid \leqslant 1\right\}\right)$, and the boundary conditions

$$
\begin{gathered}
x(0)=(0,1)^{\prime}, \\
x(1)=(1.75,2)^{\prime} .
\end{gathered}
$$

In this example $c(F, \psi)=x_{2}^{2} \psi_{1}+2 x_{1} \psi_{2}+\max \left\{\left|\psi_{1}\right|,\left|\psi_{2}\right|\right\}, \quad \frac{\partial c}{\partial x}=\left(2 \psi_{2}, 2 x_{2} \psi_{1}\right)^{\prime}$. Put $z_{1}=(1,1)^{\prime}$, then $x_{1}=(t, 1+t)^{\prime}$.

By formula (3) we have

$$
\begin{gathered}
\ell\left(\psi, z_{1}, t\right)=\psi_{1}+\psi_{2}-(1+t)^{2} \psi_{1}-2 t \psi_{2}-\max \left\{\left|\psi_{1}\right|,\left|\psi_{2}\right|\right\}= \\
=\left(1-(1+t)^{2}\right) \psi_{1}+(1-2 t) \psi_{2}-\max \left\{\left|\psi_{1}\right|,\left|\psi_{2}\right|\right\} .
\end{gathered}
$$

One can see, that the point on the 13 th iteration (with precision up to the 4 th sign)

$$
\begin{gathered}
x_{1}^{*}(t):=x_{13 \_1}=\left\{\begin{array}{l}
-0.0833 t^{4}+0.1666 t^{3}+1.3332 t, \quad t \in[0,0.4901], \\
0.8103+5.3677 t^{4}-12.5804 t^{3}+ \\
+11.8697 t^{2}-3.7173 t, \quad t \in[0.4901,1],
\end{array}\right. \\
x_{2}^{*}(t):=x_{13 \_2}=\left\{\begin{array}{l}
1+0.045 t^{5}+0.0833 t^{4}-0.1666 t^{3}+0.417 t, \quad t \in[0,0.4901], \\
1.5777+2.2506 t^{4}-5.2491 t^{3}+ \\
+6.3034 t^{2}-2.8826 t, \quad t \in[0.4901,1],
\end{array}\right.
\end{gathered}
$$

is the solution of this example. (The first index denotes the iteration number, and the second index denotes the coordinate number.) 
Rewrite inclusion (21) in the following way:

$$
\begin{gathered}
\dot{x}_{1}(t)=x_{2}^{2}(t)+u_{1}(t), \\
\dot{x}_{2}(t)=2 x_{1}(t)+u_{2}(t), \\
\left|u_{1}(t)\right|+\left|u_{2}(t)\right| \leqslant 1, \quad t \in[0,1] .
\end{gathered}
$$

Figure 3 shows the functions $x_{1}^{*}(t)$ and $x_{2}^{*}(t)$ (on the 13th iteration). As we can see, condition (23) is satisfied. Figure 3 also shows the function $\left|u_{1}^{*}(t)\right|+\left|u_{2}^{*}(t)\right|$, where $u_{1}^{*}(t)=z_{1}^{*}(t)-x_{2}^{* 2}(t), u_{2}^{*}(t)=z_{2}^{*}(t)-2 x_{1}^{*}(t)$ (on the 13th iteration). As $\left|u_{1}^{*}(t)\right|+\left|u_{2}^{*}(t)\right| \leqslant 1$ at every $t \in[0,1]$, we see, that inclusion (21) is satisfied. (Condition (22) is automatically satisfied at every iteration.) Figure 4 shows the functions $x_{1}(t)$ and $x_{2}(t)$ (on the 1st, 5 th and 9 th iterations). (The first index denotes the iteration number, and the second index denotes the coordinate number.) As it was noted, the point $z_{1}(t)=(1,1)^{\prime}$ was taken on the first iteration (so $x_{1}(t)=(t, 1+t)^{\prime}$ by $\left.(22)\right)$. Table shows the values of $I\left(z_{k}\right)$, $\left\|\nabla \varphi\left(z_{k}\right)\right\|,\left\|\nabla \chi\left(z_{k}\right)\right\|$ (on the 1st, the 5th, the 9 th and the 13th iterations). As we can see, the exact solution $\left(I\left(z_{13}\right)=0\right)$ was obtained for this example. (This is due to the structure of functional $I(z)$ and the fact, that is was possible to obtain the exact value of $\gamma_{k}$ on some iterations (including the 13th iteration) in problem (15)).
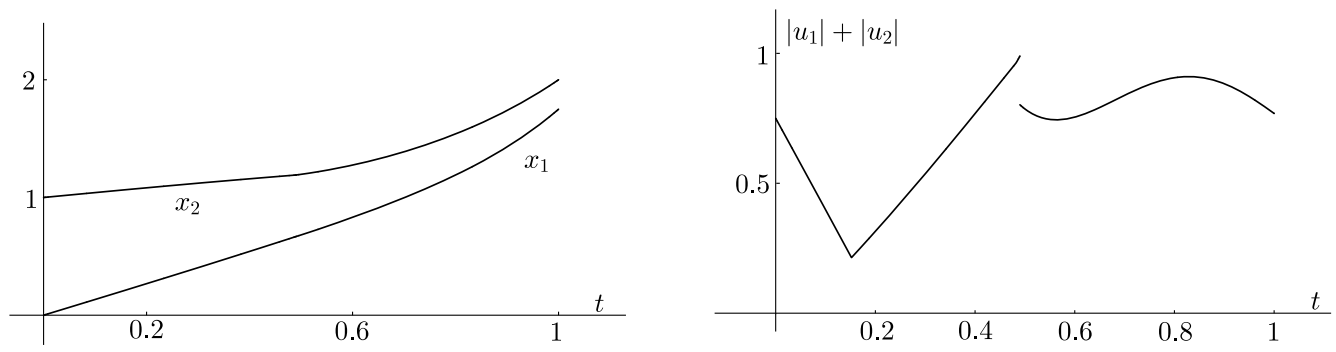

Figure 3. Solution of Example 4

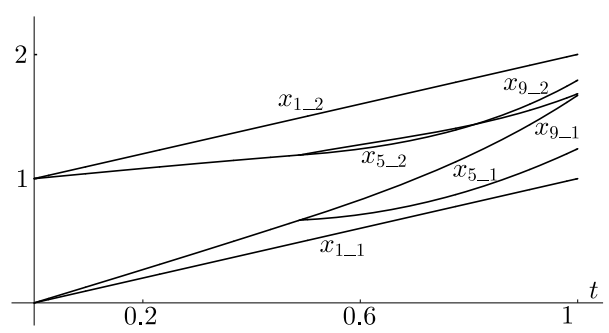

Table. Example 4: values on some iterations

\begin{tabular}{|c|c|c|c|}
\hline $\mathrm{k}$ & $\mathrm{I}\left(\mathrm{z}_{k}\right)$ & $\left\|\nabla \varphi\left(z_{k}\right)\right\|$ & $\left\|\nabla \chi\left(z_{k}\right)\right\|$ \\
\hline & & & \\
1 & 0.7089 & 1.7403 & 0.75 \\
5 & 0.3753 & 0.7286 & 0.5995 \\
9 & 0.1229 & 0.2966 & 0.2223 \\
13 & 0.0 & 0.0 & 0.0 \\
\hline
\end{tabular}

Figure 4. Example 4: some iterations

8. Conclusion. The considered problem of constructing an optimal control in the form of Lagrange with integral restriction on control reduces to the variational problem of minimizing a nonsmooth functional on the whole space. For this functional the subdifferential and the hypodifferential are obtained, the necessary minimum conditions are found, which are also sufficient in a partial case. The methods of the subdifferential descent and the hypodifferential descent are applied to the problem. The results are illustrated with numerical examples. 


\section{References}

1. Aubin J.-P., Cellina A. Differential inclusions. Berlin, Springer-Verlag Publ., 1984, 344 p.

2. Polovinkin E. S. Mnogoznachnyj analiz i differencialnye vklyucheniya [Multivalued analysis and differential inclusions]. Moscow, Fizmatlit Publ., 2014, 597 p. (In Russian)

3. Blagodatskih V. I. Vvedenie v optimalnoe upravleniye [Introduction to optimal control]. Moscow, Vysshaya shkola Publ., 2001, 239 p. (In Russian)

4. Blagodatskih V. I., Filippov A. F. Differencialnye vklyucheniya i optimalnoe upravlenie. Differential inclusions and optimal control. Proceedings of the Steklov Institute of Mathematics, 1985, vol. 169, pp. 194-252. (In Russian)

5. Filippov A. F. Differencialnye uravneniya s razryvnoj pravoj chastyu [Differential equations with discontinuous right-hand side]. Moscow, Nauka Publ., 1985, 226 p. (In Russian)

6. Watbled F. On singular perturbations for differential inclusions on the infinite interval. Journal of Mathematical Analysis and Applications, 2005, vol. 310, no. 2, pp. 362-378.

7. Gama R., Smirnov G., Watbled F. Stability and optimality of solutions to differential inclusions via averaging method. Set-Valued and Variational Analysis, 2014, vol. 22, no. 2, pp. 349-374.

8. Chengi Yi. Existence of solutions for a class of nonlinear evolution inclusions with nonlocal conditions. Journal of Optimization Theory and Applications, 2014, vol. 162, no. 1, pp. 13-33.

9. Sandberg M. Convergence of the forward Euler method for nonconvex differential inclusions. SIAM J. Numer. Anal., 2008, vol. 47, no. 1, pp. 308-320.

10. Bastien J. Convergence order of implicit Euler numerical scheme for maximal monotone differential inclusions. Z. Angew. Math. Phys., 2013, vol. 64, pp. 955-966.

11. Beyn W-J., Rieger J. The implicit Euler scheme for one-sided Lipschitz differential inclusions. Discrete and Continuous Dynamical Systems. Series B, 2010, vol. 14, no. 2, pp. 409-428.

12. Lempio F. Modified Euler methods for differential inclusions. Set-Valued Analysis and Differential Inclusions. A Collection of Papers resulting from a Workshop held in Pamporovo. Bulgaria, September 17-21, 1990. Eds by A. B. Kurzhanski, V. M. Veliov. Progr. Systems Control Theory 16. Boston, Basel, Berlin, Birkhauser Verlag Publ., 1993, pp. 131-148.

13. Veliov V. Second order discrete approximations to strongly convex differential inclusions. Systems Control Lett., 1989, no. 13, pp. 263-269.

14. Taubert K. Dierenzenverfahren fiir Schwingungen mit trockener und zdher Reibung und fiir Regelungssysteme. Numer. Math., 1976, no. 26, pp. 379-395.

15. Nikol'skiy M. S. Ob approksimacii mnozhestva dostizhimosti dlya upravlyaemogo processa [On approximation of the attainability domain of control process]. Mat. Zametki [Mat. notes], 1987, no. 41, pp. 71-76. (In Russian)

16. Nikol'skiy M. S. Ob odnom metode approksimacii mnozhestva dostizhimosti dlya differencialnogo vklyucheniya [On a method for approximation of attainable set for a differential inclusion]. Journal of Vych. Mat. Math. Phys., 1988, vol. 28, pp. 1252-1254. (In Russian)

17. Panasyuk A. I. Equations of attainable set dynamics. Pt 1. Integral funnel equations. Journal of Optim. Theory Appl., 1990, no. 64, pp. 349-366.

18. Baier R., Gerdts M., Xausa I. Approximation of reachable sets using optimal control algorithms. Numer. Algebra Control Optim., 2013, vol. 3, pp. 519-548.

19. Dontchev A., Lempio F. Difference methods for differential inclusions: A survey. SIAM Rev., 1992, vol. 34, iss. 2, pp. 263-294.

20. Schilling K. An algorithm to solve boundary value problems for differential inclusions and applications in optimal control. Numer. Funct. Anal. and Optimiz., 1989, vol. 10, iss. 7, pp. 733-764.

21. Bonnans J. F., Shapiro A. Perturbation analysis of optimization problems. New York, Springer Science+Business Media, 2000, 600 p.

22. Demyanov V. F. Usloviya ehkstremuma i variacionnoe ischislenie [Extremum conditions and variation calculus]. Moscow, Vysshaya shkola Publ., 2005, 335 p. (In Russian)

23. Kantorovich L. V., Akilov G. P. Funkcionalnyj analiz [Functional analysis]. Moscow, Nauka Publ., 1977, 741 p. (In Russian)

Received: April 8, 2018.

Accepted: September 25, 2018.

Author's information:

Alexander V. Fominyh - PhD in Physics and Mathematics, Assistant; alexfomster@mail.ru 


\title{
Метод решения дифференциальных включений с закрепленным правым концом
}

\author{
A. В. Фоминых
}

Санкт-Петербургский государственный университет, Российская Федерация, 199034, Санкт-Петербург, Университетская наб., 7-9

Для цитирования: Fominyh A. V. A method for solving differential inclusions with fixed right end // Вестник Санкт-Петербургского университета. Прикладная математика. Информатика. Процессы управления. 2018. Т. 14. Вып. 4. С. 302-315. https://doi.org/10.21638/11702/ spbu10.2018.403

В статье изучается дифференциальное включение с заданным выпуклым непрерывным многозначным отображением. Для заданного конечного промежутка времени требуется построить решение дифференциального включения, которое удовлетворяет начальному условию или обоим начальному и конечному условиям. С помощью опорных функций исходная задача сводится к задаче глобальной минимизации некоторого функционала в пространстве кусочно-непрерывных функций. В случае непрерывности производной опорной функции многозначного отображения по фазовым переменным этот функционал дифференцируем по Гато. В работе для данного функционала найдены градиент Гато, выписаны необходимые (и для некоторых частных случаев) достаточные условия минимума. На основании таких условий к исходной задаче применяется метод наискорейшего спуска. Численные методы иллюстрируют реализацию построенного алгоритма.

Ключевые слова: дифференциальные включения, опорная функция, метод наискорейшего спуска.

Контактная информация:

Фоминых Александр Владимирович - канд. физ.-мат. наук, ассистент; alexfomster@mail.ru 